\title{
PRÁTICAS DE CUIDADO EM SAÚDE MENTAL NA ATENÇÃO PRIMÁRIA À SAÚDE: REVISÃO INTEGRATIVA DE LITERATURA
}

\author{
Vandressa Lopes da Silva1, Ivan Gilson Silva Moura², Andressa Dias de Andrade Fontes \\ Amorim $^{3}$, Mariane Costa Santos de Tavares ${ }^{4}$, Bruna Batistone Bertachi ${ }^{5}$, Caroline Braga \\ Palacio $^{6}$, Sidnei Fernando Ponciano Costa Gonçalves ${ }^{7}$, Karoline de Oliveira Rodrigues ${ }^{8}$, Tamires \\ Batista pedreira ${ }^{8}$, Tâmara Campos Xavier Rodeiro ${ }^{8}$, Eduardo Fernandes da Silva ${ }^{8}$, Leonardo \\ Sousa Gomes ${ }^{8}$, Vanessa Ramalho Correia ${ }^{8}$
}

${ }^{1}$ Discente do Curso de Graduação em Medicina. Faculdades Santo Agostinho, Vitória da Conquista - BA; ${ }^{2}$ Médico Psiquiatra. Docente do Curso de Graduação em Medicina. Faculdades Santo Agostinho, Vitória da Conquista BA; ${ }^{3}$ Médica. Docente do Curso de Graduação em Medicina. Faculdades Santo Agostinho, Vitória da Conquista BA; ${ }^{4}$ Fisioterapeuta. Discente do Curso de Graduação em Medicina. Faculdades Santo Agostinho, Vitória da Conquista - BA; ${ }^{5}$ Médica. Especialista em Atenção Básica pela Universidade Federal do Paraná - UFPR; ${ }^{6}$ Médica. DSEI Alto Rio Solimões, Pólo Base Belém do Solimões; 7Médico. Atenção Primária à Saúde Guarapuava - PR;

8Discente do Curso de Graduação em Medicina, Faculdades Santo Agostinho, Vitória da Conquista - BA

\section{ARTICLE INFO}

\section{Article History:}

Received $19^{\text {th }}$ January, 2021

Received in revised form

$26^{\text {th }}$ February, 2021

Accepted $17^{\text {th }}$ March, 2021

Published online $28^{\text {th }}$ April, 2021

\section{Key Words:}

Saúde Mental, Atenção Primária à Saúde. Cuidado, Matriciamento.

*Corresponding author:

Vandressa Lopes da Silva,

\begin{abstract}
Objetivo: Discutir sobre as práticas de cuidado em saúde mental no âmbito da Atenção Primária à Saúde. Métodos: Revisão integrativa de literatura nos periódicosindexados à Scientific Eletronic Library Online (SciELO) e da Literatura Latino-Americana e do Caribe em Ciências da Saúde (Lilacs). Considerou-se estudos originais, publicados nos últimos dez anos (2011-2021), com acesso irrestrito ao conteúdo. Resultados: Foram estruturadas duas categorias temáticas: Assistência à Saúde mental e Papel do CAPS; Matriciamento em Saúde Mental. Considerações finais: A articulação entre a Rede de Atenção Psicossocial e a Atenção Básica é essencial para a constituição de um conjunto de referências capazes de acolher a pessoa em sofrimento mental e prevenir de doenças, alcançando resultados importantes para a saúde coletiva. A melhor qualificação da equipe básica, longitudinalidade do cuidado, e estímulo à participação social foram referidos como eixos norteadores da qualidade assistencial.
\end{abstract}

Copyright (C) 2021, Vandressa Lopes da Silva et al. This is an open access article distributed under the Creative Commons Attribution License, which permits unrestricted use, distribution, and reproduction in any medium, provided the original work is properly cited.

Citation: Vandressa Lopes da Silva, Ivan Gilson Silva Moura, Andressa Dias de Andrade Fontes Amorim, Mariane Costa Santos de Tavares, Bruna Batistone Bertachi, Caroline Braga Palacio, Sidnei Fernando Ponciano Costa Gonçalves, Karoline de Oliveira Rodrigues, Tamires Batista pedreira, Tâmara Campos Xavier Rodeiro, Eduardo Fernandes da Silva, Leonardo Sousa Gomes, Vanessa Ramalho Correia, 2021. "Práticas de cuidado em saúde mental na atenção primária à saúde: revisão integrativa de literatura", International Journal of Development Research, 11, (04), 46416-46419.

\section{INTRODUÇÃO}

O movimento da reforma sanitária que teve seu início no final da década de 1970 construiu as bases do Sistema único de Saúde (SUS) criando as condições de possibilidade para que a saúde se tornasse direito de todos e dever do Estado, segundo Carvalho e Passos (2015). Este processo ocorreu a partir de mudanças nos modelos de atenção e gestão nas práticas de saúde, defesa da saúde coletiva, equidade na oferta dos serviços, e protagonismo dos trabalhadores e usuários dos serviços de saúde nos processos de gestão e produção de tecnologias de cuidado (BRASIL, 2005). A construção do SUS rompeu com o caráter meritocrático que caracterizava a assistência à saúde no Brasil até a Constituição de 1988, e determinou a incorporação da saúde, como direito, numa ideia de cidadania, que considera não apenas o ponto de vista de direitos formais, de direitos políticos, mas principalmente a ideia de uma democracia substancial, de direitos substantivos, que envolviam certa igualdade de bem-estar, segundo Menicucci (2014). Nesse contexto, a Reforma Psiquiátrica Brasileira, contemporânea à reforma sanitária, foi constituída a partir do paradigma da desinstitucionalização por meio da reabilitação e da reintegração psicossocial da pessoa mentalmente adoecida. Neste modelo, os modos como elas são tratadas foram transformados, e o objeto de tratamento deixou de ser a doença e passou a ser a existência. As primeiras ações se caracterizaram pela diminuição das internações em hospitais psiquiátricos e pela criação de recursos assistenciais de caráter extra-hospitalar, de acordo com Cavalheri 
(2010). Sendo assim, é na década de 90, marcada pelo compromisso firmado pelo Brasil na assinatura da Declaração de Caracas e pela realização da II Conferência Nacional de Saúde Mental, que passam a entrar em vigor no país as primeiras normas federais regulamentando a implantação de serviços de atenção diária, fundadas nas experiências dos primeiros CAPS, NAPS e Hospitais-dia, e as primeiras normas para fiscalização e classificação dos hospitais psiquiátricos (BRASIL, 2005). É no contexto da promulgação da lei 10.216 e da realização da III Conferência Nacional de Saúde Mental, que a política de saúde mental do governo federal alinhada com as diretrizes da Reforma Psiquiátrica passa a consolidar-se, ganhandomaior sustentação e visibilidade. Linhas específicas de financiamento são criadas pelo Ministério da Saúde para os serviços abertos e substitutivos ao hospital psiquiátrico e novos mecanismos são criados para a fiscalização, estão e redução programada de leitos psiquiátricos no país (BRASIL, 2005). Neste mesmo período, o processo de desinstitucionalização de pessoas longamente internadas é impulsionado, com a criação do Programa "De Volta para Casa". Uma política de recursos humanos para a Reforma Psiquiátrica é construída, e é traçada a política para a questão do álcool e de outras drogas, incorporando a estratégia de redução de danos (BRASIL, 2005).

Desta forma, a III Conferência consolida a Reforma Psiquiátrica como política de governo e confere aos CAPS o valor estratégico para a mudança do modelo de assistência, defende a construção de uma política de saúde mental para os usuários de álcool e outras drogas, e estabelece o controle social como garantia do avanço da Reforma Psiquiátrica no Brasil. É a III Conferência Nacional de Saúde Mental, com ampla participação dos movimentos sociais, de usuários e de seus familiares, que fornece os substratos políticos e teóricos para a política de saúde mental no Brasil (BRASIL, 2005). Paralelo a isso, o desenvolvimento da Estratégia Saúde da Família nos últimos anos marca um progresso indiscutível da política do SUS. Atendendo ao compromisso da integralidade da atenção à saúde, o Programa Saúde da Família (PSF), criado na década de 90, vem investindo na promoção da saúde da população e na prevenção de doenças, alcançando resultados importantes para a saúde coletiva. Estruturado em equipes de Atenção Básica, a cobertura do Programa já alcança todo o país (BRASIL, 2005). Diante do exposto, a atual rede de atenção à saúde mental brasileira é parte integrante do Sistema Único de Saúde (SUS), ela é composta por Centros de Atenção Psicossocial (CAPS), Serviços Residenciais Terapêuticos (SRT), Centros de Convivência, Ambulatórios de Saúde Mental e Hospitais Gerais, caracteriza-se por ser essencialmente pública, de base municipal e com um controle social fiscalizador e gestor no processo de consolidação da Reforma Psiquiátrica (BRASIL, 2005).

Para Alves (2001), citado por citado por Quinderé, Jorge e Franco (2014), um dos dispositivos estratégicos mais eficientes para a superação do modelo manicomial são os Centros de Atenção Psicossocial. Eles são serviços destinados a articular as ações de saúde mental em rede e junto à Estratégia de Saúde da Família (ESF), ambulatórios, leitos de internação em hospitais gerais e ações de suporte e reabilitação psicossocial (ONOCKO-CAMPOS; FURTADO, 2006), citado por Quinderé, Jorge e Franco. (2014). Com o desenvolvimento da Estratégia Saúde da Família (ESF) houve um progresso na política do SUS. A ESF é composta por equipes multiprofissionais, e o seu campo de intervenção são as pessoas, famílias e suas relações com a comunidade e com o meio ambiente. A realidade destas equipes de Atenção Básica vem demonstrando que, cotidianamente, elas se deparam com problemas de saúde mental, segundo Brasil (2005). Nesse sentido, da aproximação que se estabeleceu entre as equipes da ESF e os dispositivos de saúde mental, prioritariamente os CAPS e os Ambulatórios, decorreu um novo arranjo no trabalho e nos fluxos de uma rede de atenção psicossocial que tomou a ESF como mais um recurso para a saúde mental. Santos e Souza (2014) apontam para importância de se discutir a produção de cuidado e saberes em saúde mental no contexto da saúde da família, sobretudo nos últimos dez anos, no Brasil, já que a atenção voltada à saúde mental é relativamente nova e existem muitas dúvidas relacionadas aos sujeitos envolvidos na saúde mental, necessitando assim de uma melhor abordagem da produção de cuidado e saberes em saúde mental no contexto da saúde da família que tem como objetivos solucionar, melhorar, organizar e transformar a saúde mental obtida pelo SUS. De acordo com Casanova e Oliveira (2009) o fortalecimento do cuidado a partir do campo das relações interpessoais com uma escuta dialógica entre os profissionais e comunidade é importante para a consolidação da assistência no SUS. Desse modo, a presença do diálogo permite a compreensão das necessidades de saúde colocadas pela população, e a partir delas, desenvolver ações, integrando saberes e práticas das diversas áreas. Em tal contexto, o objetivo deste estudo foi discutir sobre as práticas de cuidado em saúde mental no âmbito da Atenção Primária à Saúde.

\section{METóDOS}

A discussão apresentada neste constructo configura-se como uma revisão integrativa de literatura nos periódicos indexados à Scientific Eletronic Library Online (SciELO) e da Literatura Latino-Americana e do Caribe em Ciências da Saúde (Lilacs). Foram utilizados para busca os descritores em ciências da saúde (DECS): Atenção primária á Saúde; Saúde Mental; Matriciamento. Considerou-se estudos originais, publicados nos últimos dez anos (2011-2021), com acesso irrestrito ao conteúdo. A análise dos dados se deu a partir da abordagem qualitativa, culminando com o surgimento de duas categorias temáticas: "Assistência à Saúde Mental e Papel do CAPS"; "Matriciamento em Saúde Mental".

\section{RESULTADOS E DISCUSSÃO}

Assistência à Saúde Mental e Papel do CAPS : A Atenção à Saúde Mental, embora considerada essencial ainda possui grandes obstáculos a serem transpostos. Muitos autores ressaltam a importância da integralidade do cuidado e inclusão de usuários e serviços de forma integrada. No Brasil, o descompasso entre a magnitude da demanda e a oferta de serviços de saúde mental, além da baixa incorporação de especialistas para lidar com pacientes graves, constituem-se em uma ameaça à sustentabilidade do modelo decuidadoàsaúdementalnaAPS (HIRDES, 2018). Dessa forma, o diálogo entre as equipes da Atenção Básica e o conhecimento do funcionamento da Rede de Atenção Psicossocial é a base para um fluxo de atendimento em saúde mental de qualidade. Entretanto, esse cuidado ainda mantém obstáculos na assistência, visto que ainda hoje os profissionais não estão adequadamente capacitados, demonstrando ainda pouco domínio sobre o que realizar no campo da saúde mental na Atenção Básica (CARVALHO et al., 2018). Nessa conjuntura, o cuidado inclusivo deve ser perpassado pela dimensão clínica, uma vez ela orienta uma direção de tratamento. Desse modo, O CAPS, assim como o ambulatório e os demais dispositivos de saúde mental têm um potencial clínico legítimo na rede de cuidados ampliados e inclusivos(DAUMOUS; ERLICH, 2017). Os mesmos autores pontuam que da aproximação que se estabeleceu entre as equipes da ESF e os dispositivos de saúde mental, prioritariamente os CAPS e os Ambulatórios, decorreu um novo arranjo no trabalho e nos fluxos de uma rede de atenção psicossocial que tomou a ESF como mais um recurso para a saúde mental. Essa reorganização visa ao compartilhamento de responsabilidades entre os serviços de saúde mental e os da atenção primária(DAUMOUS; ERLICH, 2017).

Para promover a integralidade do cuidado e inclusão dos sujeitos, outro pilar essencial seria a maior participação da sociedade. Todavia, segundo Santos e Souza (2014) a participação social dos sujeitos e familiares também é incipiente o que dificulta a percepções dos determinantes sociais para esse grupo de vulnerabilidade, tendo como consequências projetos terapêuticos verticalizados que não atuam na lógica da reabilitação social de fortalecer as potencialidades dos sujeitos. A culpabilização dos familiares é um fenômeno presente em no estudo de Campos et.al (2011), nele os trabalhadores identificam os familiares como um grupo afastado do serviço. Compartilhando da mesma ideia, os relatos dos profissionais dos estudos de Contatinidis 
(2017) apontam que alguns familiares têm dificuldades em assumir o cuidado de seu parente com transtorno mental. Isso se dá, principalmente, pelo pensamento de segregação, vestígios da cultura manicomial que ainda se faz presente. Esse é um dos impasses da atenção psicossocial, que ao procurar manter o portador de transtornos mentais em comunidade, deve compartilhar responsabilidade com os familiares. Mesmo em contextos adversos, ressalta-se que a APS consegue, ao estruturar vínculo com usuários e familiares potencializar a resolutividade do cuidado. A APS atua na lógica da longitudinalidade do cuidado. Quando tal longitudinalidade é quebrada, seja pela falha no fluxo assistencial, ou pela troca dos profissionais da equipe a qualidade do cuidado e resolutividade são profundamente impactados. Em Campos et. al (2011) os profissionais apontaram que grande número de contratos temporários também é um fator que leva à alta rotatividade de profissionais da saúde, principalmente médico. Ainda, nesse viés, na pesquisa de Miranda et al. (2014) e Marftum et al. (2016) os profissionais mencionaram que a rotatividade da equipe com a mudança de governoatrapalhanaformaçã devínculoe confiançacomospacientes. Repercutindo em uma assistência à Saúde mental empobrecida. Fernandes, Frazão e Rocha (2014) e Medina, Neto e Hirdes (2014), em seus estudos ressaltam a necessidade de maior investimento, principalmente na capacitação dos trabalhadores da Atenção Básica para a assistência em Saúde Mental. Logo, carências no preparo para atuação em equipe, desconhecimento do papel desta equipe na assistência ao paciente e nos benefícios que este modelo traz ao serviço geram prejuízos à assistência ambulatorial no que diz respeito ao elo entre o paciente e o profissional tão importante na adesão ao tratamento, e na consolidação dos princípios e diretrizes doSUS.

Matriciamento em Saúde mental: Segundo Campos e Domitti (2017) o Apoio Matricial (AM) tem como objetivo oferecer retaguarda assistencial e suporte técnico-pedagógico a equipes de referências da Atenção Básica. Contudo, com relação ao apoio matricial dentro da rede, os sujeitos compreenderam como sendo uma alternativa de assistência realizada através do encaminhamento aos serviços de saúde mental, frente a um usuário queprocura a ESF. Diferentemente da compreensão errônea de alguns serviços e profissionais o apoio matricial em saúde mental é o eixo principal que sustenta a parceria deste campo com a atenção primária, pois oferece um suporte técnico especializado às equipes da ESF e proporciona uma educação permanente, sem contudo, representar apenas um serviço para referenciamento de usuários (BONFIM et al., 2013). As equipes da Atenção Básica se apresentam como um recurso estratégico para o enfrentamento dos problemas de saúde mental. Contudo, nem sempre a Atenção Básica apresenta condições para tal enfrentamento, por isso, o apoio matricial a essas equipes garante a responsabilização compartilhada dos casos e exclui a lógica do encaminhamento, pois visa aumentar a capacidade resolutiva de problemas de saúde pela equipe local (CHIAVERINI, 2011). Azevedo, Gondim e Silva (2013) perceberam nos discursos uma confusão no entendimento do termo, já que o matriciamento muitas vezes é interpretado como "transferência de responsabilidade" de serviço para serviço, sendo assim, no âmbito da ESF, isto pode acontecer devido ao despreparo dos profissionais da equipe em lidar com esta demanda, considerando a forte influência do modelo biomédico desaúde. Já nos estudos de Hirdes (2018), a fala de um dos sujeitos evidenciou que ocorre a personalização das relações, a corresponsabilização e a cogestão do processo, demonstrando que o descompasso varia de acordo com a equipe e localidade.
Além disso, o trabalho em equipe interdisciplinar pode apresentar desafios no que tange os conflitos interpessoais, falta de comunicação e de articulação. Medina, Neto e Hirdes (2014) ressaltam a despreparação dos médicos para realização do apoio matricial. Em algumas regiões os conflitos entre equipes fazem com que os serviços acumulemtarefas exercendo funçõe squenãosãodesua responsabilidade, e dificulte oprocessod ecuidadointegral ao usuário. Em Marftum et. al (2016) o desconhecimento do funcionamento dos dispositivos que compõem a rede de saúde mental por parte dos profissionais do ambulatório e por outros profissionais que compõem a rede de saúde foi citado como um aspecto preocupante para a continuidade do tratamento do paciente com transtorno mental. Além disso, foi ressaltada a falta de comunicação interna e a ausência da reunião geral no serviço e, ainda de acordo com os participantes, os próprios profissionais do ambulatório desconhecem as atividades que são realizadas pelos outros trabalhadores no serviço e em outros pontos da rede de assistência. Para corrigir esta falha, além das reuniões gerais da equipe, a troca de informações interna e entre os serviços deve ser precisa e contínua para que o cuidado não seja prejudicado. Neste contexto, a Educação Permanente em Saúde para os profissionais que compõem esta rede é aliada para a mudança desse cenário. Entretanto a implantação, expansão e qualificação do apoio matricial é um desafio para a gestão da política nacional de saúde mental, e a garantia de acessibilidade à atenção um dos maiores desafios do Sistema Único de Saúde (SUS).

\section{CONCLUSÃO}

Nos estudos considerados verificou-se que a APS se configura como um eixo ordenador do cuidado em saúde mental, possuindo papel essencial na integralidade do cuidado. Alguns obstáculos ainda persistem para maior resolutividade deste nível assistencial como fragmentação da rede, alta rotatividade de profissionais, bem como a compreensão equivocada do dispositivo de matriciamento, que acaba funcionando apenas na lógica de encaminhamento dos usuários. Necessidade de maior investimento na qualificação da equipe de saúde também foi um ponto bastante abordado nos estudos selecionados.

\section{REFEÊNCIAS}

AZEVEDO, D. M; GONDIM, M. C. S. M; SILVA, D. S. S. Apoio matricial em saúde mental: percepção de profissionais no território. Revista de Pesquisa Cuidado é Fundamental online. Rio de Janeiro, v.5, n.1, p. 33113311, jan/mar. 2013. Disponível em: <https://www. redalyc.org/pdf/5057/505750897016.pdf $>$. Acesso em: 04 jun. 2020.

BONFIM, Iris Guilherme et al . Apoio matricial em saúde mental na atenção primária à saúde: uma análise da produção científica e documental. Interface (Botucatu), Botucatu, v. 17, n. 45, p. 287-300, June 2013.

BRASIL. Ministério da Saúde. Secretária de Atenção à Saúde. DAPE. Coordenação Geral de Saúde Mental. Reforma psiquiátrica e política de saúde mental no Brasil. In: Conferência Regional de Reforma dos Serviços de Saúde Mental: 15 anos depois de Caracas. 2005, Brasília. OPAS. Brasília, 2005. P. 6-25. Disponível em:

CAMPOS G. W. S; DOMITTI A.C. Apoio matricial e equipe de referência: uma metodologia para gestão do trabalho 
interdisciplinar em saúde. Caderno de saúde pública. Rio de Janeiro, v.23, n.2, p. 399-407, fev. 2007. Disponível em:

CAMPOS ONOCKO, R. et. al. Saúde mental na atenção primária à saúde: estudo avaliativo em uma grande cidade brasileira. Ciência \& saúde coletiva, vol.16, n.12, p. 46434652, Rio de Janeiro Dez. 2011. Disponível em:

CARVALHO, Maria de Fátima Alves Aguiar et al . Desarticulação da rede psicossocial comprometendo a integralidade do cuidado. Rev. esc. enferm. USP, São Paulo, v. 51, e03295, 2018

CHIAVERINI, D.H. (Org.). Guia prático de matriciamento em saúde mental. Ministério Saúde: Centro de Estudos e Pesquisa em Saúde Coletiva, 2011. Disponível em:

CONSTANTINIDIS, T.C. Profissionais de saúde mental e familiares de pessoas com sofrimento psíquico: encontro ou desencontro?. Psicologia USP. São Paulo,v.28, n.1, jan/abr. 2017. Disponível em: <http://www.scielo.br/pdf/ pusp/v28n1/16785177- pusp-28-01-00023.pdf $>$. Acesso em: 07 jun. 2020.

DAMOUS, I. ; ERLICH, H. O ambulatório de saúde mental na rede de atenção psicossocial: reflexões sobre a clínica e a expansão das políticas de atenção primária. Physis Revista de Saúde Coletiva, Rio de Janeiro, vol. 27, n.4, p. 911932,2017. Disponível em: <https://www.scielo.br/pdf/ physis/v27n4/0103-7331-physis-27- 04-00911.pdf $>$. Acesso em: 04 jun. 2020.

ELIA, L. A rede da atenção na saúde mental - articulações entre CAPS e ambulatórios. In: BRASIL. MINISTÉRIO DA SAÚDE. Caminhos para uma política de saúde mental infanto-juvenil. 2005, p. 49-61.

FERNANDES, M. J; ROCHA, H. A; FRAZÃO, D. P. S. Articulações em saúde: contribuições para o fortalecimento do trabalho em rede entre centro de atenção psicossocial e estratégia de saúde da família. Revista de Saúde Pública do SUS/MG. Belo Horizonte, vol.2, n.1, p.55-70, jul/dez. 2014. Disponível em:

GARLA, C.C.; FUREGATO, A.R.F; SANTOS. J.L. F. Profissionais de ambulatórios de saúde mental: perfil, práticas e opiniões sobre as políticas. Cadernos Brasileiros de Saúde Mental. Florianópolis, vol.2, n.4-5, p.74-93, ago. 2010. Disponível em:

MAFTUM, M.A et al. Ambulatório de saúde mental: fragilidades apontadas por profissionais. Texto e Contexto Enfermagem. Florianópolis, v.23, n.3, 2016. Disponível em: <http://www.scielo.br/pdf/tce/v25n3/pt_0104-0707tce-25-03-0040014.pdf >. Acesso em: 04 jun. 2020.

MARCONI, Marina de Andrade; LAKATOS, Eva Maria. Fundamentos de Metodologia Científica. Entrevista. 5. ed. - São Paulo: Atlas, 2003. p. 197.

MENDES, K. D. S; SILVEIRA, R. C. C. P; GALVÃO, C. M. Revisão integrativa:
MENICUCCI, T. M. G. História da reforma sanitária brasileira e do Sistema Único de Saúde: mudanças, continuidades e a agenda atual. História, Ciencias, Saúde - Manguinhos. Rio de Janeiro, v.21, n.1, p.77-92, jan/mar. 2014. Disponível em:

método de pesquisa para a incorporação de evidências na saúde e na enfermagem. Texto e Contexto Efermagem. Florianópolis, vol.17, n.4,p.758-764, out/dez. 2008. Disponível em: $\quad<$ http://www.scielo.br/pdf/tce/ v17n4/18.pdf $>$. Acesso em: 04 jun. 2020.

Ministério da Saúde. Saúde mental no SUS: os Centros de Atenção Psicossocial. Brasília: Ministério da Saúde; 2004.

MIRANDA et al. Estudo de uma Rede de Atenção Psicossocial: Paradoxos e efeitos da precariedade. Psicologia: ciência e profissão. Brasília, vol.34, n.3, p.592-611, 24 jul/set. 2014. Disponível em: $<$ https://www. scielo.br/pdf/pcp/v34n3/1982-3703-pcp-34- 03-0592.pdf $>$. Acesso em: 04 jun. 2020.

NETO, M. R. G; MADINA, T.S.S.; HIRDES,A. Apoio matricial em saúde mental na percepção dos profissionais especialistas. Aletheia. Canoas, n.45, dez. 2014. Disponível em: <http://pepsic.bvsalud.org/ scielo.php?script $=$ sci_arttext\&pid $=$ S1413$03942014000200011>$. Acesso em: 04 jun. 2020.

OLIVEIRA, C.M; CASANOVA, A.O. Vigilância da saúde no espaço de práticas da atenção básica. Ciência e saúde coletiva. Rio de Janeiro, vol.14, n.3, mai/jun 2009. Disponível em: <http://www.scielo.br/ scielo.php?script $=$ sci_arttext\&pid $=\mathrm{S} 1413$ 81232009000300029>. Acesso em: 04 jun. 2020.

PASSOS, E; CARVALHO, Y.M. A formação para o SUS abrindo caminhos para a produção do comum. Saúde e sociedade. São Paulo, v. 24, n.1, p.92-101, abr/jun. 2015. Disponível em: <http://www.scielo.br/pdf/ sausoc/v24s1/0104-1290-sausoc-24- s1-00092.pdf $>$. Acesso em: 04 Jun. 2020.

QUINDERÉ, P.H.D; JORGE, M.S.B; FRANCO, T. B. Rede de Atenção Psicossocial: qual o lugar da saúde mental? Physis Revista de Saúde Coletiva, Rio de Janeiro, vol. 24, n. 1, p. 253-271, 2014. Disponível em:

SANTOS, R.C; SOUZA, J.E. Construção Social da aprendizagem em saúde mental e saúde da família. Ilheus, BA. Editus, 2014, 216 p.. Disponível em:

SILVA JUNIOR, A G. Modelos Tecnoassistenciais em Saúde: o debate no campo da saúde coletiva. São Paulo. HUCITEC, 2006

SPINK, M.J; MEDRADO, B. Produção de sentidos no cotidiano: uma abordagem teórico-metodológica para análise das práticas discursivas. In: SPINK, M.J. (Org.). Práticas discursivas e produção de sentidos no cotidiano: aproximações teóricas e metodológicas. São Paulo: Cortez, 2013. p.41-61.

TRIVIÑOS, A. N. S. Introdução à pesquisa em ciências sociais: a pesquisa qualitativa em educação. São Paulo: Atlas, 1987. 